\title{
Missing heritability, next-generation genome-wide association studies and primary cancer prevention: an Atlantean illusion?
}
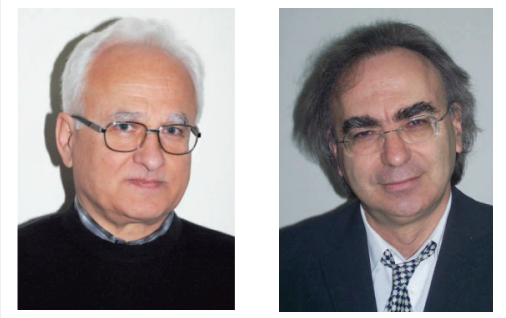

“... faster and cheaper massively parallel sequencing technology has changed our understanding of human biology and disease."

Christos Katsios' \& Dimitrios H Roukos ${ }^{\dagger 1,2}$

'Department of Surgery, loannina University School of Medicine, loannina, TK 451 10, Greece

2Personalized Cancer Networks Medicine, Biobank, loannina University, loannina, TK 451 10, Greece †Author for correspondence: Tel.: +30 2651007423 - Fax: +30 2651007094 -droukos@uoi.gr

When a draft of the complete human genome sequence was announced in 2000 , governments, funders, industry and researchers made grand promises about how genome-based discoveries would revolutionize science. Indeed, 10 years later, faster and cheaper massively parallel sequencing technology has changed our understanding of human biology and disease. Now, these structural and functional genome data reveal that life and diseases, such as cancer, are much more heterogeneous and complex than we anticipated. Using this advanced technology, genome-wide association studies (GWAS) have identified a flood of novel genes and mutations. However, these association studies can explain only approximately $20-25 \%$ of traits or diseases. In this article, we discuss how this 'missing heritability' can be explained, and the perspectives and challenges to overcome this missing heritability by using next-generation sequencing technology for the next-generation GWAS in order to complete the catalogue of genetic variants for each cancer type. Whether this biomedical research revolution could be translated into medical progress to predict and prevent cancer in individual persons will also be explored.

Atlantis is a legendary island described in Plato's dialogues Timaeus and Critias. In Plato's account, Atlantis was a naval power lying "in front of the Pillars of Hercules" that conquered many parts of western Europe and Africa approximately $9600 \mathrm{BCE}$. After a failed attempt to invade Athens, Atlantis sank into the ocean "in a single day and night of misfortune." Some scholars dispute the existence of Atlantis, while others insist that Plato took inspiration from

contemporary events such as the destruction of Helike in 373 BCE. Alan Cameron states: "It is only in modern times that people have taken the Atlantis story seriously; no one did so in antiquity" [1]. Even now, Atlantis inspires today's literature, from science fiction to comic books to films. Similarly to how the mystery of Atlantis may never be solved, modern GWAS have failed to explain the missing heritability of cancer $[2,3]$. It is difficult to predict whether this missing heritability will be verified or whether it will remain a mystery.

\begin{abstract}
"It is estimated that among persons with a significant family history of breast cancer, only $20-25 \%$ of familial cancer risk can be explained by candidate cancer genes. How could this 'missing heritability' be explained?"
\end{abstract}

\section{Primary prevention of cancer}

Most cancer types, particularly when they are diagnosed in more advanced stages, still remain incurable; therefore, prevention is always better than treatment. However, the medical progress in the prevention setting has been slow over recent decades. The best measure of the effectiveness of the primary prevention strategy is cancer incidence and mortality rates. In the published global cancer burden landscape from 2011, cancer is the leading cause of death in economically developed countries, and the burden of cancer is increasing in economically developing countries [4]. The rise in cancer incidence in developing countries is thought to be a result

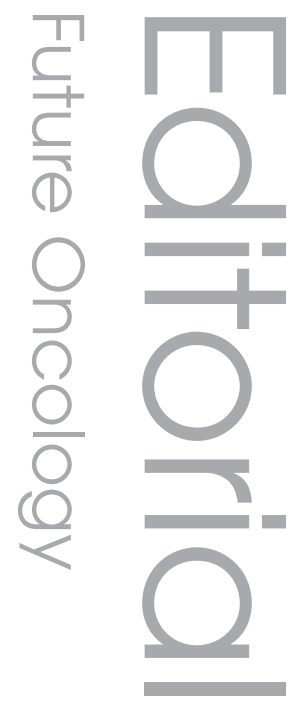


of the increased adoption of cancer-associated lifestyle choices including smoking, physical inactivity and 'westernized' diets [4].

To achieve a drastic reduction of cancer incidence, an understanding of the molecular mechanisms underlying tumorigenesis appears to be essential. Tumorigenesis-based development of biomarkers for identifying high-risk individuals before the manifestation of clinical symptoms is a major goal of current and emerging research. However, these efforts, with a few exceptions, have not been translated into the clinical setting.

Cancer arises from the accumulation of genetic and epigenetic alterations. The environment and heritability are responsible for somatic and inherited mutations, respectively, while multiple epigenetic modifications are often detected within malignant tumors [5]. There are two paradigms of isolated success regarding nongenetic and genetic risk factors. Tobacco smoking control has reduced the incidence and mortality of lung cancer in the USA and several other developed countries [4]. Genetic testing for identifying high-risk women carrying heritable mutations in the BRCA1/2 genes can limit breast cancer development by prophylactic surgery or surveillance [6]. However, both lifestyle and genetic factors, namely smoking and BRCA1/2 mutations, increase the risk of lung and breast cancer by 20 -fold, respectively, and thus it is easy to isolate and identify these factors by epidemiological and genetic linkage studies. These factors, with very large effects on disease, are the exception. However, pathogenesis of common diseases is mostly multifactorial and very complex, suggesting major challenges in primary prevention.

\section{Secondary prevention}

Given the very good prognosis and high cure rates for patients with early diagnosis when the cancer is still localized, efforts for screening programs in several developed countries have yielded increased rates of early-stage cancer detection and improved survival rates [4]. However, clinicians are now more and more concerned by overdetection, overdiagnosis and overtreatment of diseases as a result of sensitive tests. For example, PSA screening levels for early prostate cancer detection have little impact on mortality reduction rates, and treatments received as a result can increase adverse effects on quality of life [7,8]. Mammographic screening for early breast cancer detection has been considered to be a major advance in reducing mortality from breast cancer. However, the more recent Norwegian trial has demonstrated only modest mortality benefits, raising questions regarding the current utility of mammographic screening [9].

\section{Rare, high-penetrance, inherited mutations \& prevention of hereditary cancer syndromes}

Heritable mutations in specific single genes have substantial effects on cancer risk, causing hereditary cancer syndromes in the vast majority of individuals carrying these mutations. The genetic cause of these 'Mendelian' diseases can be revealed by sequencing the single gene that is responsible for a specific cancer development. Conventional linkage studies in high-risk families with several family members affected by specific types of cancer have linked inherited mutations with cancer risk. Carriers of germ-line mutations in BRCA1 or BRCA2, in mismatch repair genes (MLH1, MSH2, MSH6 and PMS2) and the $C D H 1$ (E-cadherin) gene face a high lifetime risk of up to $75 \%$ for developing hereditary breast/ovarian cancer, nonpolyposis colorectal cancer and diffuse gastric cancer syndrome, respectively $[6,10,11]$.

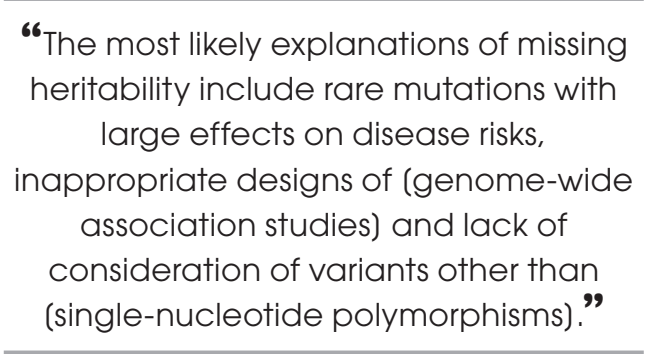

Family histories and genetic testing permit the identification of these high-risk individuals carrying mutations that cause cancer. Preventive interventions are now available and include prophylactic surgery and surveillance of these high-risk mutation carriers. Several studies with risk-reducing surgery in mutation carriers have provided evidence for a more than $90 \%$ reduction in both the risk of developing a specific cancer type and mortality from the disease among carriers who underwent prophylactic surgery [12]. Surveillance can detect cancer at an early stage in some high-risk mutation carriers and enable better quality of life than preventive surgery, but it can often fail, delaying diagnosis and increasing mortality rates. Therefore, although 25\% of mutation carriers will not develop cancer in their life, most expert teams suggest bilateral salpingo-oophorectomy and/or bilateral mastectomy for BRCA mutation carriers, colorectal 
cancer resection and, in females, an additional total hysterectomy for mismatch repair mutation carriers and total gastrectomy for $\mathrm{CDH} 1$ mutation carriers, despite their considerable adverse effects on quality of life [12].

Despite advances in genetic testing being more widely available, it can identify less than 7 or $3 \%$ of all breast [6] and colorectal [10] or gastric cancer [12] patients, respectively, who are affected by these single genes that cause cancers each year. It has become obvious that for the remaining $93-97 \%$ of cases, much more sophisticated research strategies will be required.

\section{'Missing heritability' \& genome-wide association studies}

Over the past 5 years, GWAS have begun to scour human genetic samples for the signals of individual variations. Typically, such studies assess hundreds of thousands of genetic variants in thousands of individuals sorted by traits - a certain height, obesity or various disorders [13]. Using single-nucleotide polymorphisms (SNPs) developed via technological advances with genome sequencing, the present generation of GWAS have used SNP microarrays containing between 50,000 and up to 1 million SNPs to screen populations for significant differences. Although a wealth of novel SNPs and genes has been identified, there has been a clinical disappointment in the assessment of small risks of these newly discovered variants, despite statistically significant differences observed [13]. In a recent pooled analysis from several published GWAS, it was calculated that 201 SNPs associated with height could explain approximately $16 \%$ of the genetic variance, 142 SNPs that are associated with Crohn's disease could explain approximately $20 \%$ and 67 SNPs could explain approximately $17 \%$ of the genetic variance in each of three common cancers [14]. In the clinic, the first efforts to improve risk prediction by adding the new genetic variants to established conventional breast cancer risk factors have recently failed [15-17]. It is estimated that among persons with a significant family history of breast cancer, only $20-25 \%$ of familial cancer risk can be explained by candidate cancer genes $[7,16]$. How could this 'missing heritability' be explained?

\section{Explaining \& potentially overcoming 'missing heritability'}

Generally, associations between SNPs and traits or disease tend to be of modest effect size, with a median odds ratio per copy of the risk allele of $1.33[13,14]$. The most likely explanations of missing heritability include rare mutations with large effects on disease risks, inappropriate designs of GWAS and lack of consideration of variants other than SNPs. More recently, the concept of tumorigenesis driven by genome function and cancer networks, rather than alterations in genome structure, has attracted wider attention $[13,18]$.

Most GWAS have used genotyping platforms with less than 1 million SNPs, without consideration of copy-number variants or genomic rearrangements. These studies were designed to detect SNPs at a frequency of more than 5\% in the population. If there are rare variants with substantial effects, it is not surprising that these rare mutations could not be captured by the available association studies [13].

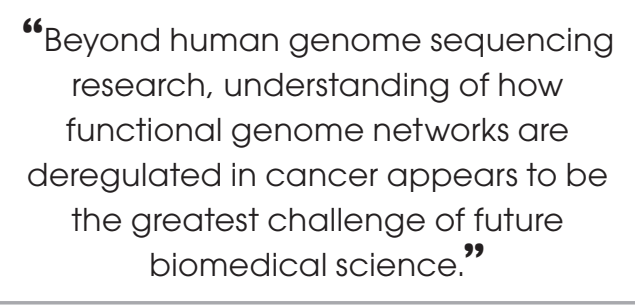

To overcome these challenges, vendors such as Illumina (CA, USA) and Affymetrix (CA, USA) will develop assays with 5 million SNPs in the next year [3] that are based on more recently released data with a much larger number of genetic variants provided by the HapMap 3 project [19]. By using these microchips in larger sample sizes, rarer mutations at frequencies of $0.5 \%$ could be detected by next-generation GWAS. However, we should await these new GWAS data to assess whether such rare mutations with large effects on cancer risk exist or whether other mechanisms (e.g., functional deregulation of the genome) drive tumorigenesis. Important insights into structural aberrations and functional deregulation in the cancer genome will be investigated in the International Cancer Consortium project [20] using next-generation sequencing platforms. This project will provide the largest cancer genetic variation database, which could lead to novel genomics-based classifications, new biomarkers and the development of new drugs and could be used as the basis of reference for future, more sophisticated approaches [20].

\section{Conclusion}

Since the identification of hereditary cancer syndromes by conventional linkage studies and the genetic testing-based selection of high-risk 


\section{Bibliography}

1. Cameron A: Greek Mythography in the Roman World. Oxford University Press, UK, 124 (2004)

2. Manolio TA, Collins FS, Cox NJ: Finding the missing heritability of complex diseases. Nature 461(7265), 747-753 (2009).

3. Baker M: Genomics: the search for association. Nature 467(7319), 1135-1138 (2010).

4. Jemal A, Bray F, Center MM, Ferlay J, Ward E, Forman D: Global cancer statistics. CA Cancer J. Clin. 61(2), 69-90 (2011).

5. Lander ES: Initial impact of the sequencing of the human genome. Nature 470(7333), 187-197 (2011).

6. Narod SA, Offit K: Prevention and management of hereditary breast cancer. J. Clin. Oncol. 23(8), 1656-1663 (2005).

7. Hudson T: Genome-sequencing anniversary. Genomics and clinical relevance. Science 331(6017), 547 (2011).

8. Strope SA, Andriole GL: Prostate cancer screening: current status and future perspectives. Nat. Rev. Urol. 7(9), 487-493 (2010).
9. Welch HG: Screening mammography a long run for a short slide? N. Engl. J. Med. 363(13), 1276-1278 (2010).

10. Lynch HT, Lynch PM, Lanspa SJ, Snyder CL, Lynch JF, Boland CR: Review of the Lynch syndrome: history, molecular genetics, screening, differential diagnosis, and medicolegal ramifications. Clin. Genet. 76(1), 1-18 (2009).

11. Guilford P, Hopkins J, Harraway J et al:: E-cadherin germline mutations in familial gastric cancer. Nature 392(6674), 402-405 (1998).

12. Guillem JG, Wood WC, Moley JF et al.; ASCO; SSO: ASCO/SSO review of current role of risk-reducing surgery in common hereditary cancer syndromes. J. Clin. Oncol. 24(28), 4642-4660 (2006).

13. Manolio TA: Genomewide association studies and assessment of the risk of disease. N. Engl. J. Med. 363(2), 166-176 (2010).

14. Park JH, Wacholder S, Gail MH et al.: Estimation of effect size distribution from genome-wide association studies and implications for future discoveries. Nat. Genet. 42(7), 570-575 (2010). promise against for preventing cancer rather than curing patients with advanced disease in the future.

\section{Financial \& competing interests disclosure}

The authors have no relevant affiliations or financial involvement with any organization or entity with a financial interest in or financial conflict with the subject matter or materials discussed in the manuscript. This includes employment, consultancies, honoraria, stock ownership or options, expert testimony, grants or patents received or pending, or royalties.

No writing assistance was utilized in the production of this manuscript.

15. Travis RC, Reeves GK, Green J et al.; Million Women Study Collaborators: Geneenvironment interactions in 7610 women with breast cancer: prospective evidence from the Million Women Study. Lancet 375(9732), 2143-2151 (2010); erratum in: Lancet 375(9732), 2142 (2010).

16. Narod SA: Genes, the environment, and breast cancer. Lancet 375(9732), 2123-2124 (2010).

17. Wacholder S, Hartge P, Prentice R et al: Performance of common genetic variants in breast-cancer risk models. N. Engl. J. Med. 362(11), 986-993 (2010).

18. Green ED, Guyer MS; National Human Genome Research Institute: Charting a course for genomic medicine from base pairs to bedside. Nature 470 (7333), 204-213 (2011).

19. International HapMap 3 Consortium; Altshuler DM, Gibbs RA, Peltonen L et al.: Integrating common and rare genetic variation in diverse human populations. Nature 467(7311), 52-58 (2010).

20. Hudson TJ, Anderson W, Artez A et al: International network of cancer genome projects. Nature 464, 993-998 (2010). 